\title{
Green's Functions from Quantum Cluster Algorithms
}

\author{
R. Brower ${ }^{\mathrm{a}, \mathrm{c}}, \mathrm{S}$. Chandrasekharan $\mathrm{fb}^{\mathrm{b}, \mathrm{c}}$ and U.-J. Wiese ${ }^{\mathrm{c}}$ \\ a Department of Physics \\ Boston University \\ Boston MA 02215, USA \\ a T-8, Theoretical Division \\ Los Alamos National Laboratoy \\ Los Alamos, NM 87545 \\ ${ }^{c}$ Center for Theoretical Physics \\ Laboratory for Nuclear Science and Department of Physics \\ Massachusetts Institute of Technology \\ Cambridge MA 02139, USA \\ Preprints \\ MIT-CTP 2686 \\ LA-UR 98-91
}

PACS: 02.70.Lq, 05.30.-d, 05.50.+q, 11.15.Ha

Keywords: Quantum Spins, Clusters, Green's Functions, Improved Estimators

\begin{abstract}
We show that cluster algorithms for quantum models have a meaning independent of the basis chosen to construct them. Using this idea, we propose a new method for measuring with little effort a whole class of Green's functions, once a cluster algorithm for the partition function has been constructed. To explain the idea, we consider the quantum XY model and compute its two point Green's function in various ways, showing that all of them are equivalent. We also provide numerical evidence confirming the analytic arguments. Similar techniques are applicable to other models. In particular, in the recently constructed quantum link models, the new technique allows us to construct improved estimators for Wilson loops and may lead to a very precise determination of the glueball spectrum.
\end{abstract}

\footnotetext{
${ }^{1}$ This work is supported in part by funds provided by the U.S. Department of Energy (D.O.E.) under cooperative research agreement DE-FC02-94ER40818.

2 Address after Jan, 1998: Physics Department, Duke University, Durham NC 27708.
} 


\section{Introduction}

Cluster Algorithms have had a tremendous success in numerically solving some interesting field theoretic models [1, 2]. Such algorithms have the potential to drastically reduce the problem of critical slowing down, and perhaps even eliminate it completely. The Swendsen-Wang [3] algorithm was the first cluster algorithm to be invented. It has been extended to both classical [4] and quantum spin systems [5, 6] with continuous symmetries. On the other hand, the success of cluster algorithms has been limited to spin models without frustration. Further, it has not been possible to construct an efficient cluster algorithm for Wilson's formulation of lattice gauge theory. However, a new formulation of gauge theories, proposed recently, provides a possible alternative. The corresponding quantum link models [7] are a quantum version of Wilson's gauge theory, in the same sense as the quantum Heisenberg model is a quantum version of the 2-d $O(3)$ model. A cluster algorithm for the simplest $U(1)$ quantum link model has recently been constructed [8]. While in quantum spin models the clusters are closed loops, in quantum link models they are world-sheets of electric flux strings. It is expected that similar algorithms exist for other gauge groups. Thus, it appears that cluster algorithms can also play an important role in the numerical solution of gauge theories.

Updating a system with an efficient cluster algorithm accelerates its evolution through configuration space. However, in quantum models this does not automatically imply that one can efficiently measure various Green's functions. For example, one is interested in creation and annihilation events described by operators that are not diagonal in the basis in which the path integral is constructed. The measurement of such "quantum flip" operators appears to be very difficult. In fact one appears to be restricted to a very limited class of Green's functions built from the diagonal operators. For example, let us consider the quantum XY model expressed in terms of the Pauli matrices $\sigma_{x}^{i}$ at the lattice sites $x$. When we work in the basis where $\sigma_{x}^{3}$ is diagonal, Green's functions for products of $\sigma_{x}^{3}$ operators can be measured easily. On the other hand, it appears that Green's functions involving $\sigma_{x}^{1}$ or $\sigma_{x}^{2}$ are difficult - if not impossible - to measure. A measurement of $\sigma_{x}^{1}$ or $\sigma_{x}^{2}$ in the path integral described by eigenstates of $\sigma_{x}^{3}$ necessarily "flips" the state of the corresponding

quantum spin. In other words, Green's functions of $\sigma_{x}^{1}$ or $\sigma_{x}^{2}$ receive contributions from superselection sectors of the theory, which are not sampled by a conventional Monte Carlo algorithm which simply simulates the partition function in a particular basis.

For quantum cluster algorithms, there is a remarkable way out of this impasse. By virtue of the auxiliary percolation process used in a quantum cluster Monte Carlo algorithm, the requisite information for all quantum Green's functions can in fact be extracted. In this paper, we formulate a method, which we call "quantum flip" measurements, for computing non-trivial Green's functions from a quantum spin cluster simulation. Our construction of the "quantum flip" measurement pro- 
cedure is based on a generalization of the classical Fortuin-Kasteleyn [9] mapping to quantum clusters and on the observation that a basis-independent description exists for the resulting quantum random cluster model. For the Swendsen-Wang cluster algorithm, it is well known that the Fortuin-Kasteleyn mapping demonstrates the equivalence between the partition function of the Ising or Potts model with the partition function of an associated random cluster model. This step, which is sometimes ignored in discussions of cluster algorithms, leads to a purely geometric procedure to measure spin correlations. For quantum spin models our generalized Fortuin-Kasteleyn map naturally also leads to a systematic and rigorous method to measure any operator or Green's function. The quantum Green's functions involving operators non-diagonal in a particular cluster update operation are related to cuts (or "flips") which cleave the original cluster into disjoint sub-clusters. Based on this picture, it is possible to apply similar ideas to more complicated systems such as gauge theories expressed as quantum link models. In qualitative terms a similar suggestion was made in ref. [10]. Our "quantum flip" algorithm gives a simple explanation of the idea and provides a general approach to measure any Green's function.

The article is organized as follows. In section 2, we reformulate the quantum spin model as a model that describes the dynamics of clusters with internal quantum degrees of freedom. We use the quantum XY model as an example. The reformulation demonstrates the basis-independence of the quantum clusters. In section 3 we show how one can measure any operator using the cluster algorithm. In section 4 , we illustrate how the observations of sections 2 and 3 are realized in the quantum $\mathrm{XY}$ model, by constructing two cluster algorithms which are related by a change of basis. We show why the cluster properties are independent of the basis, and provide numerical evidence confirming the analytic results. In section 5 we suggest how to use similar ideas in the $U(1)$ quantum link model to obtain improved estimators for Wilson loops and present our conclusions.

\section{Basis-Independence of Clusters}

In this section, we show that constructing a cluster algorithm for a quantum spin model is equivalent to rewriting the path integral in terms of geometrical objects, which we call quantum clusters, with internal quantum degrees of freedom, in this case quantum spins. We then argue that the dynamics within each cluster can be written in a form which is invariant under basis transformation of the quantum spin Hilbert space. In the next section, we discuss how these observations lead to a method for measuring a whole class of operators. In order to illustrate the idea we consider the two-dimensional quantum XY model. Most of the observations extend trivially to other models including quantum link models. 
The quantum XY model is described by the Hamilton operator

$$
H=\sum_{x, \hat{\mu}} h_{x, \hat{\mu}} ; \quad h_{x, \hat{\mu}}=\frac{J}{2}\left(\sigma_{x}^{1} \sigma_{x+\hat{\mu}}^{1}+\sigma_{x}^{2} \sigma_{x+\hat{\mu}}^{2}\right) .
$$

Here $\hat{\mu}=1,2, \ldots, d$, denotes spatial directions and $x$ denotes the position on an $L^{d}$ lattice. The partition function is given by

$$
Z=\operatorname{Tr} \exp (-\beta H)=\operatorname{Tr} \prod_{i=1}^{N} \exp (-\epsilon H) .
$$

In the last step we have introduced the Suzuki-Trotter discretization such that $\epsilon N=$ $\beta$. It should be noted that it is not necessary to discretize time. For quantum systems in a discrete basis the path integral is well-defined in continuous time, and can be simulated directly in the time-continuum [11]. In this paper we work at discrete time, but all results have a direct continuous-time version. In the above form the partition function is not yet accessible by numerical simulations. In fact, the term $\exp (-\epsilon H)$ needs to be expanded further. The basic idea is to write the Hamiltonian as a sum of interactions arranged in a checker board pattern such that a minimal number of spins interact in a single time step. For example, in $d=2$, one decomposes

$$
H=H_{1}+H_{2}+H_{3}+H_{4} .
$$

This divides the Hamiltonian into four terms in a checker board pattern with

$$
H_{1}=\sum_{x \in(2 m, n)} h_{x, \hat{1}}, \quad H_{2}=\sum_{x \in(m, 2 n)} h_{x, \hat{2}}, \quad H_{3}=\sum_{x \in(2 m+1, n)} h_{x, \hat{1}}, \quad H_{4}=\sum_{x \in(m, 2 n+1)} h_{x, \hat{2}} .
$$

Every $H_{i}$ contains a sum of commuting operators, each of which represents a two-spin interaction. Thus $\exp \left(-\epsilon H_{i}\right)$ can be computed easily, and evolves all the spin states through a single time step. The partition function is then defined on a hypercubic lattice with $4 N$ time slices. It is equal to the trace over the Hilbert space of a product of $2 N L^{2}$ transfer matrices connecting pairs of spins. This is because the spins interact in pairs in a checker board pattern depending on which of the $\exp \left(-\epsilon H_{i}\right)$ is active in a particular time step. There are $V / 2$ two-spin interactions, where $V=4 N L^{2}$ is the volume of the lattice. In the $d=1$ case, one only needs two terms in the expansion of the Hamiltonian, i.e., $H=H_{1}+H_{2}$. Then there are $2 \mathrm{~N}$ time slices and the partition function is a trace of a product constructed from $N L$ transfer matrices, connecting pairs of spins. A typical lattice for $d=1$ is shown in figure 1. Generalization to higher dimensions is straight forward.

The transfer matrix that evolves a pair of spins through a single time step is denoted by an operator $T$. Since $T$ represents a two-spin interaction, it is equivalent to a $4 \times 4$ plaquette matrix. Denoting the basis for the spins by $|s\rangle$, the operator $T$ can be expanded as

$$
T=\sum_{s_{1}, s_{2}, s_{3}, s_{4}}\left|s_{3} s_{4}\right\rangle \mathcal{T}\left(s_{1}, s_{2}, s_{3}, s_{4}\right)\left\langle s_{1} s_{2}\right|
$$


where $s_{1}, s_{2}$ are the spins in one time slice and $s_{3}, s_{4}$ are the same spins in the next time slice. For example, if we choose the basis to be eigenstates of $\sigma_{x}^{3}$, the non-zero matrix elements of $T$ are given by

$$
\begin{aligned}
& \mathcal{T}(++++)=\mathcal{T}(----)=1, \\
& \mathcal{T}(+-+-)=T(-+-+)=\cosh (\epsilon J), \\
& \mathcal{T}(+--+)=T(-++-)=\sinh (\epsilon J) .
\end{aligned}
$$

Here \pm represent the eigenstates of $\sigma^{3}$ with eigenvalues \pm 1 . This leads to the path integral form for the partition function,

$$
Z=\operatorname{Tr}\left(\prod_{p=1}^{V / 2} T_{p}\right)=\sum_{\mathcal{S}} \mathcal{W}\left[s_{i}\right]
$$

where of course the product over operators $T_{p}$ for each of the $V / 2$ plaquettes must be appropriately "time" ordered. In the last step, we have replaced the trace by a sum over spin configurations $\mathcal{S}$, which is a collection of spins $s_{i}$ located at the space-time points of a $(d+1)$-dimensional lattice, weighted by the weight $\mathcal{W}$. The associated probability distribution of the spin configuration is given by $\mathcal{P}\left[s_{i}\right]=\mathcal{W}\left[s_{i}\right] / Z$. The weight function is a product over the four-spin plaquette interactions, which takes the form

$$
\mathcal{W}\left[s_{i}\right]=\mathcal{T}\left(s_{1}, s_{2}, s_{3}, s_{4}\right) \mathcal{T}\left(s_{4}, s_{5}, s_{6}, s_{7}\right) \ldots \mathcal{T}\left(s_{V-3}, s_{V-2}, s_{V-1}, s_{V}\right)
$$

Due to the trace structure of the partition function, each spin state $s_{i}$ occurs in two different $\mathcal{T}$ operators. The sum over a particular spin $s_{i}$ makes the partition function independent of the basis that is used to describe that spin state. We will see that the basis-independence of the partition function extends to the clusters. One can rewrite it completely in terms of clusters without referring to the basis of the Hilbert space. This observation also leads to similar definitions for the expectation values of operators.

We now proceed with the construction of a cluster algorithm and the FortuinKasteleyn map onto its associated "random cluster" model. The construction of a cluster algorithm is equivalent to rewriting $\mathcal{T}\left(s_{1}, s_{2}, s_{3}, s_{4}\right)$ as a sum over products of simpler tensors [12]. For example, we can expand

$$
\mathcal{T}\left(s_{1}, s_{2}, s_{3}, s_{4}\right)=a_{0} \mathcal{M}_{0}\left(s_{1}, s_{2}, s_{3}, s_{4}\right)+a_{1} \mathcal{M}_{1}\left(s_{1}, s_{2}, s_{3}, s_{4}\right)+a_{2} \mathcal{M}_{2}\left(s_{1}, s_{2}, s_{3}, s_{4}\right),
$$

as a sum of three terms, where we selected a minimal tensor basis,

$$
\mathcal{M}_{0}=\delta_{s_{1}, s_{3}} \delta_{s_{2}, s_{4}}, \mathcal{M}_{1}=\delta_{s_{1}, s_{4}} \delta_{s_{2}, s_{3}}, \mathcal{M}_{2}=\sigma_{s_{1}, s_{2}}^{1} \sigma_{s_{3}, s_{4}}^{1}
$$

with the respective weights uniquely determined to be

$$
a_{0}=\frac{1}{2}(1+\exp (-\epsilon J)), a_{1}=\frac{1}{2}(1-\exp (-\epsilon J)), a_{2}=\frac{1}{2}(\exp (\epsilon J)-1) .
$$


Thus each tensor $\mathcal{T}$ in the partition function is equivalent to a sum of three tensors, $\mathcal{M}_{n}$, labeled by a plaquette percolation state, $n_{p}=0,1,2$. This allows us to rewrite the partition function as a sum over $3^{V / 2}$ terms since there are $V / 2 \mathcal{T}_{\mathrm{s}}$ in the partition function. By choosing one of the three terms for each plaquette, one is led to a joint probability distribution, $\mathcal{P}\left[s_{i} ; n_{p}\right]$, where $n_{p}$ labels the percolation state of each plaquette. Equivalently, since we have chosen a tensor decomposition in which each term is given by a matrix connecting two adjacent spins, each of the $3^{V / 2}$ terms can be represented by a graph $G$ with connected components (i.e. clusters) $\mathcal{C}_{i}, i=1, \ldots, N_{G}$. A simple example of a graph in a 1-d model model is shown in figure 1.

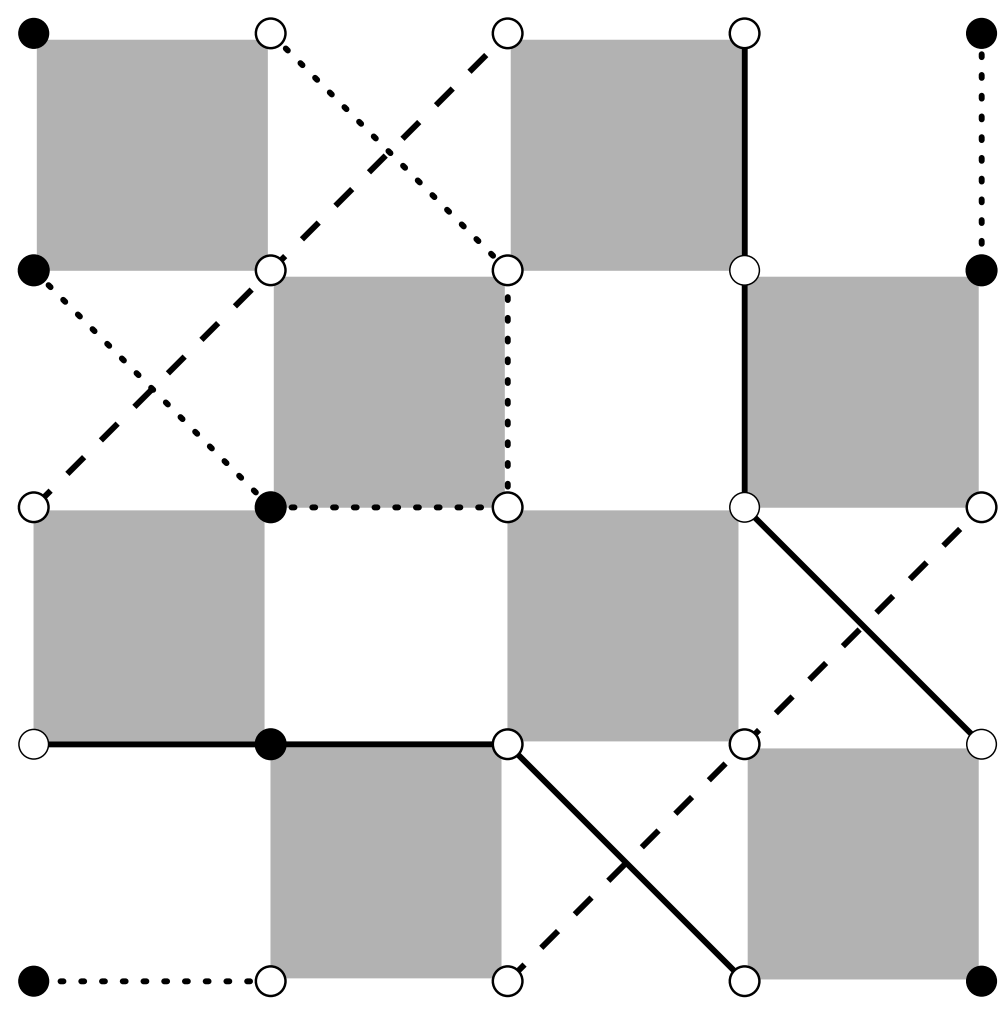

Figure 1: A typical graph in 1-d with $L=4$, and $N=2$ : Since $H=H_{1}+H_{2}$, one has $2 N$ time-slices and $V=2 N L$. Every white square represents a two-spin interaction. The graph $G$ shown here splits the lattice into three clusters, represented by a solid line, a broken line and a dotted line. It is easy to read off $w(G)=a_{0}^{2} a_{1}^{4} a_{2}^{2}$.

The Fortuin-Kasteleyn map is based on the simple observation that the joint distribution, $\mathcal{P}\left[s_{i} ; n_{p}\right]$, has two marginal distributions: One, obtained by summing over the percolation states, is the original spin probability distribution,

$$
\mathcal{P}\left[s_{i}\right]=\sum_{\left\{n_{p}\right\}} \mathcal{P}\left[s_{i} ; n_{p}\right],
$$

while the other marginal distribution, obtained by summing over the spin variables,

$$
\mathcal{P}[G]=\sum_{\mathcal{S}} \mathcal{P}\left[s_{i} ; n_{p}\right],
$$


is a new "random cluster model". The notation on the left-hand side acknowledges the fact that in our application each distribution of integer percolation states $n_{p}$ uniquely corresponds to a graph $G$.

It should also be noted that this mapping can be cast in purely operator terms for the quantum model. Going back to the operator form of the partition function, we have a plaquette operator, $T$, in eq.(5) for each four-spin matrix element, $\mathcal{T}\left(s_{1}, s_{2}, s_{3}, s_{4}\right)$. Obviously, the operator $T$ can be expressed as a sum of simpler operators, $\sum_{n} a_{n} M_{n}$ with matrix elements that reproduce the above expansion eq.(9) for $\mathcal{T}\left(s_{1}, s_{2}, s_{3}, s_{4}\right)$. The contribution to the partition function for a particular graph $G$ takes the form of a trace $\operatorname{Tr}\left[M_{G}\right]$, where the operator $M_{G}$ is the appropriate timeordered product of factors $M_{n_{p}}$ and the full partition function can be rewritten as

$$
Z=\sum_{G} w(G) \operatorname{Tr}\left[M_{G}\right]
$$

Thus we have defined the probability distribution,

$$
\mathcal{P}[G]=\frac{1}{Z} w(G) \operatorname{Tr}\left[M_{G}\right]
$$

for the associated quantum random cluster model.

The cluster algorithm is a way to realize these dynamics in a Monte Carlo method. In a particular basis it reproduces a graph $G$ with the clusters $\mathcal{C}_{i}(i=$ $\left.1, \ldots, N_{G}\right)$ with the weight $w(G)$ and internal spin orientations $\operatorname{Tr}\left[M_{G}\right]$. However, since $w(G)$ and $\operatorname{Tr}\left[M_{G}\right]$ are basis-independent, it is clear that algorithms can be constructed in more than one basis. Such algorithms produce clusters with exactly the same geometrical properties. We will illustrate this in the case of the quantum $\mathrm{XY}$ model in section 4. It is possible that the efficiency of algorithms working in different bases are different since the cluster growth dynamics would be different. Moreover, it is important to realize that in a new basis one may choose new cluster decompositions defined by eq.(9) [12 which are not equivalent to a basis change. In this case, the cluster properties of two random cluster models are in general inequivalent.

The above picture is a generalization of the well-known Fortuin-Kasteleyn mapping 99 to quantum models. Such a quantum mapping was also considered in another more technical paper [13]. Here we present a new way to understand the mapping. For example, we have shown that the quantum XY model is equivalent to a model that involves the dynamics of geometrical objects (in the present case closed loops), with internal quantum degrees of freedom (in this case spins). Summing over the spin degrees of freedom defines a partition function that describes the dynamics of the clusters independent of the basis used for the spin states. This is at the heart of the Fortuin-Kasteleyn mapping for the Potts model. As we will show in the next section, the basis-independence of the clusters brings into focus some interesting ideas on how to measure general Green's functions. In particular, 
it shows why the idea of ref. [10] works and provides a systematic way to measure any operator.

\section{Measurement of Green's Functions}

The basis-independence of the clusters suggests that it must be possible to measure any operator with a given cluster algorithm. This is indeed true. For example, in the XY model it is possible to measure the $\sigma_{x}^{1} \sigma_{y}^{1}$ correlators even when one uses the cluster algorithm in the basis where $\sigma^{3}$ is diagonal. The fact that such measurements are possible has recently been suggested in ref. [10]. Based on the above ideas, we explain how this is possible. In fact, our approach extends to all operators, although the measurement of the above correlator seems to be the simplest illustration of our approach.

The expectation value of an operator $\mathcal{O}_{x}$, acting at the site $x$, is defined by

$$
\left\langle\mathcal{O}_{x}\right\rangle=\frac{1}{Z} \operatorname{Tr}\left[\mathcal{O}_{x} \exp (-\beta H)\right]
$$

Rewriting the above expression one obtains

$$
\left\langle\mathcal{O}_{x}\right\rangle=\frac{1}{Z} \sum_{G} w_{G} \operatorname{Tr}\left[\mathcal{O}_{x} M_{G}\right] .
$$

Let us consider the steps that lead to the numerator of eq.(17). The numerator of eq.(16) can be written as a product of transfer matrices $T$ as described in the previous section. However, now there is an additional operator $O_{x}$ acting on a specific site in a particular time-slice $t$. Then there are two transfer matrices adjacent to this site. The matrix $T^{-}$connects the site with the previous time-slice, and $T^{+}$connects it with the next time-slice. The operator $\mathcal{O}_{x}$ is sandwiched between the two. In the next step each $T$ is expanded into three terms and the whole expression is again written as a sum of $3^{V / 2}$ terms. Each of the $3^{V / 2}$ terms represents a graph $G$ with clusters $\mathcal{C}_{i},\left(i=1, \ldots, N_{G}\right)$. The weights $w(G)$ are the same as before. However, due to the presence of the operator $\mathcal{O}_{x}$ the previous expression is modified. It now contains the matrix $\mathcal{O}_{x}$ appropriately sandwiched between the tensors $\mathcal{M}_{0}, \mathcal{M}_{1}$, or $\mathcal{M}_{2}$ coming from $T^{+}$and $T^{-}$. In the example of the quantum XY model, where the clusters are closed loops and $M_{G}$ is a product of $\delta_{s s^{\prime}}$ or $\left(\sigma^{1}\right)_{s, s^{\prime}}$, one simply inserts the matrix $\left(\mathcal{O}_{x}\right)_{s, s^{\prime}}$ at the right place. When the cluster turns back in time, the appropriate matrix to be inserted is $\left(\mathcal{O}_{x}^{T}\right)_{s, s^{\prime}}$. Such subtleties must be kept in mind.

It is easy to find the right prescription to measure any operator using the cluster algorithm. The cluster algorithm produces the graph $G$ with the weight,

$$
\mathcal{P}[G]=\frac{1}{Z} w(G) \operatorname{Tr}\left[M_{G}\right] .
$$


Thus we get the formula

$$
\left\langle\mathcal{O}_{x}\right\rangle=\left\langle\frac{\operatorname{Tr}\left[\mathcal{O}_{x} M_{G}\right]}{\operatorname{Tr}\left[M_{G}\right]}\right\rangle .
$$

To evaluate this expression one must perform all possible cluster flips, which can be done very efficiently using an improved estimator. As we will see in the next section, the above formula can be easily extended to measure any two-point Green's function in the quantum XY model.

\section{Two-Point Green's functions}

Here we will demonstrate the power of the above ideas by measuring various twopoint Green's functions in the quantum XY model, using the cluster algorithm in the $\sigma^{3}$ basis. We show how the dynamics of the clusters allows us to prove analytically that the $O(2)$ symmetry is maintained. We also construct a cluster algorithm in a different basis, and show how the same features emerge in the new basis.

Let us consider a cluster algorithm obtained by decomposing the transfer matrix as given in eq.(9)). The rules for cluster growth are given in figure 2. One can
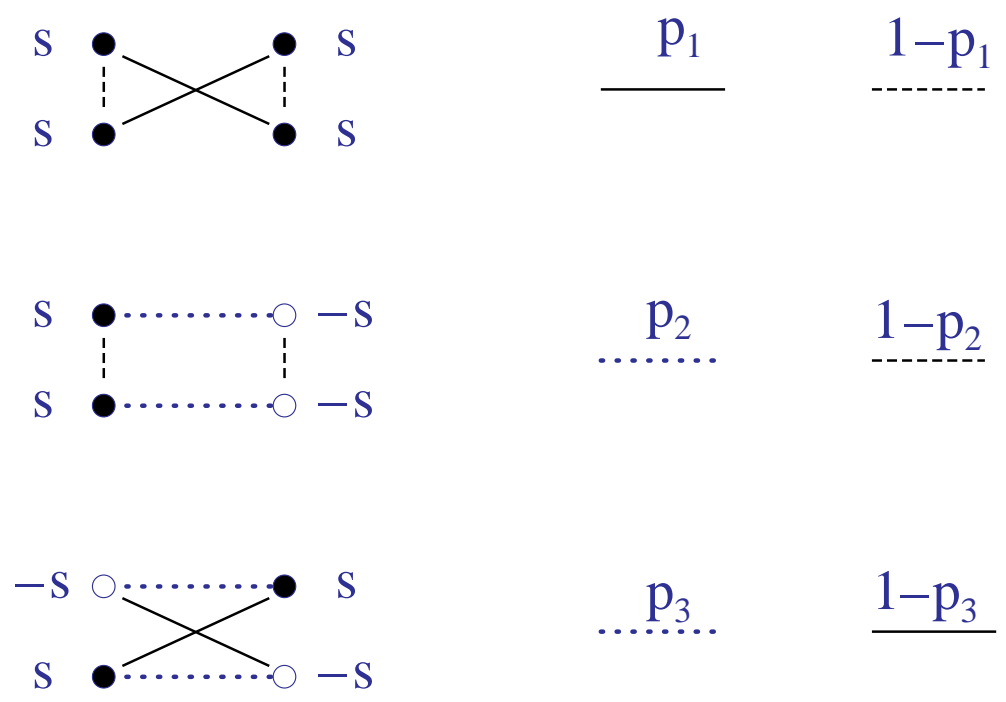

Figure 2: The rules for creating the bonds that define the graph $G$ : The sites are described by filled circles and the time axis is vertical. The spin states in the $\sigma^{3}$ basis are denoted by $s$, which can be \pm . The probabilities for choosing the bonds are $p_{1}=$ $[1-\exp (-\epsilon J)] / 2, p_{2}=[\exp (\epsilon J)-1] /[2 \cosh (\epsilon J)]$, and $p_{3}=[\exp (\epsilon J)-1] /[2 \sinh (\epsilon J)]$.

easily derive some properties of the clusters. They are closed loops, since each site is connected to two bonds, one for an interaction forward, and the other for an interaction backward in time. Further, the bonds connect spins of the same orientation when the cluster grows forward or backward in time. However, when 
they connect spins in the same time slice, they connect opposite spins. Globally, this leads to an interesting property of the clusters. If the clusters have positive spins when they grow forward in time, they have negative spins when they turn back and grow backwards in time. This property of the clusters is important for proving that the correlators $\left\langle\sigma_{x}^{1} \sigma_{y}^{1}\right\rangle$ and $\left\langle\sigma_{x}^{2} \sigma_{y}^{2}\right\rangle$ are equal.

Let us measure the correlator $\left\langle\sigma_{x}^{1} \sigma_{y}^{1}\right\rangle$ with the above cluster algorithm. Note that since $\sigma_{x}^{1}$ changes the spin state, it is a priori unclear how to measure $\left\langle\sigma_{x}^{1} \sigma_{y}^{1}\right\rangle$, given just the spin configuration. In fact, a spin configuration that contributes to the correlator will never be generated in the cluster algorithm. However, eq.(19) tells us to measure $\operatorname{Tr}\left[\sigma_{x}^{1} \sigma_{y}^{1} M_{G}\right] / \operatorname{Tr}\left[M_{G}\right]$ for each graph generated by the cluster algorithm. $]^{[}$ Each graph $G$ represents a set of clusters $\mathcal{C}_{i}\left(i=1, \ldots, N_{G}\right)$. Since $M_{G}$ is a product of $\delta_{s s^{\prime}}$ or $\sigma_{s, s^{\prime}}^{1}$, the trace contracts the spins on each cluster separately. Thus $\operatorname{Tr}\left[M_{G}\right]$ becomes a product of traces for each cluster, i.e.,

$$
\operatorname{Tr}\left[M_{G}\right]=\prod_{i=1}^{N_{G}} \operatorname{Tr}\left[M_{\mathcal{C}_{i}}\right]
$$

where $N_{G}$ is the number of clusters in $G$. Further, given one spin in the cluster, the orientations of all other spins in the cluster are determined. This means that $\operatorname{Tr}\left[M_{\mathcal{C}_{i}}\right]=2$. In other words, $\operatorname{Tr}\left[M_{G}\right]$ measures the number of configurations obtained from all possible flips of the clusters. Since each cluster contributes two configurations, $\operatorname{Tr}\left[M_{G}\right]=2^{N_{G}}$. On the other hand, $\operatorname{Tr}\left[\sigma_{x}^{1} \sigma_{y}^{1} M_{G}\right]$ is the same, except that at the sites $x$ and $y$ the spins are now flipped. Clearly, if $x$ and $y$ were in different clusters, this flip would be inconsistent going around the closed loop, which means that $\operatorname{Tr}\left[\sigma_{x}^{1} M_{\mathcal{C}_{i}}\right]=0$. However, when $x$ and $y$ are both in the same cluster, the contribution is exactly the same as $\operatorname{Tr}\left[M_{G}\right]$. Thus, each time a cluster is produced that includes both $x$ and $y$, we get a contribution 1 to the measurement of $\left\langle\sigma_{x}^{1} \sigma_{y}^{1}\right\rangle$. Due to the translational invariance of the Hamiltonian, it is possible to consider any pair of points $x, y$ within a given cluster and count it as a contribution to the correlator. In this way, it is possible to obtain the complete two-point correlation function $\left\langle\sigma_{x}^{1} \sigma_{y}^{1}\right\rangle$.

What about the $\left\langle\sigma_{x}^{2} \sigma_{y}^{2}\right\rangle$ correlator? Since the model is $O(2)$ invariant, it must be equal to $\left\langle\sigma_{x}^{1} \sigma_{y}^{1}\right\rangle$. This is indeed the case, although the proof is a bit subtle. While an insertion of $\sigma^{1}$ flips the spin, an insertion of $\sigma^{2}$ flips the spin along with a multiplication by a factor $\pm i$. This means that, when $x$ and $y$ are in different clusters, $\operatorname{Tr}\left[\sigma_{x}^{2} M_{\mathcal{C}_{i}}\right]$ is zero as before. However, if they are in the same cluster, one must figure out what the factors $\pm i$ contribute. Here we invoke a special property of the clusters to show that there is always exactly one $i$ and one $-i$ factor coming from the two $\sigma^{2}$ insertions. The point is that if $x$ and $y$ are in the branch of the cluster that grows in one particular time direction, the spin flips from $+(-)$ to $-(+)$ at the first $\sigma^{2}$ insertion and from $-(+)$ to $+(-)$ at the second insertion. This gives one

\footnotetext{
${ }^{3}$ In the interest of simplicity we ignore the fact that when the operators $\sigma_{x}^{1}$ and $\sigma_{y}$ are on different "time" slice the product $\sigma_{x}^{1} \sigma_{y}^{1} M_{G}$ should, of course, be appropriately "time" ordered.
} 
factor $i$ and one $-i$. On the other hand, if $x$ is in a branch growing in a particular time direction and $y$ is in a branch growing in the opposite direction, the spin flips from $+(-)$ to $-(+)$ at the first and from $+(-)$ to $-(+)$ at the second $\sigma^{2}$ insertion. However, since the cluster is growing backward in time at one of the insertions, the actual matrix element is that of $\left(\sigma^{2}\right)^{T}$ which again gives one $i$ and one $-i$ factor. Thus, if $x$ and $y$ are in the same cluster, the contributions to $\left\langle\sigma_{x}^{1} \sigma_{y}^{1}\right\rangle$ and $\left\langle\sigma_{x}^{2} \sigma_{y}^{2}\right\rangle$ are the same from each graph generated by the cluster algorithm.

It is straightforward to measure $\left\langle\sigma_{x}^{+} \sigma_{y}^{-}\right\rangle$or $\left\langle\sigma_{x}^{-} \sigma_{y}^{+}\right\rangle$in the same way, and one gets half of the contribution of $\left\langle\sigma_{x}^{1} \sigma_{y}^{1}\right\rangle$ for each. Thus, it is possible to measure any two-point Green's function directly using formula eq.(19). In fact, one can easily use it also to measure higher $n$-point Green's functions, which, however, get contributions from more than one cluster. It is interesting that there is a clusternotion of "connected" and "disconnected" pieces to the four-point Green's function. The connected piece comes entirely from within a cluster, while the disconnected piece comes from two separate clusters.

We now consider a change of basis and show the basis-independence of the clusters using a concrete example. One can consider a basis change given by

$$
\left|s_{x}\right\rangle=\sum_{s_{x}^{\prime}}\left(e^{-i \sigma_{x}^{1} \pi / 4}\right)_{s_{x} s_{x}^{\prime}}\left|s_{x}^{\prime}\right\rangle .
$$

This basis change can be applied to each of the spin states. In terms of the new basis, the transfer matrix given in eq.(5) changes to

$$
\begin{aligned}
\hat{T}= & \sum_{s_{1}, s_{2}, s_{3}, s_{4}} \sum_{s_{1}^{\prime}, s_{2}^{\prime}, s_{3}^{\prime}, s_{4}^{\prime}}\left(e^{-i \sigma^{1} \pi / 4}\right)_{s_{3} s_{3}^{\prime}}\left(e^{-i \sigma^{1} \pi / 4}\right)_{s_{4} s_{4}^{\prime}} \\
& \left|s_{3}^{\prime} s_{4}^{\prime}\right\rangle \mathcal{T}\left(s_{1}, s_{2}, s_{3}, s_{4}\right)\left\langle s_{1}^{\prime} s_{2}^{\prime}\right|\left(e^{i \sigma^{1} \pi / 4}\right)_{s_{1}^{\prime} s_{1}}\left(e^{i \sigma^{1} \pi / 4}\right)_{s_{2}^{\prime} s_{2}}
\end{aligned}
$$

The transformed tensor can be written as

$$
\mathcal{T}^{\prime}\left(s_{1}, s_{2}, s_{3}, s_{4}\right)=a_{0} \mathcal{M}_{0}^{\prime}\left(s_{1}, s_{2}, s_{3}, s_{4}\right)+a_{1} \mathcal{M}_{1}^{\prime}\left(s_{1}, s_{2}, s_{3}, s_{4}\right)+a_{2} \mathcal{M}_{2}^{\prime}\left(s_{1}, s_{2}, s_{3}, s_{4}\right)
$$

where

$$
\mathcal{M}_{0}^{\prime}=\delta_{s_{1}, s_{3}} \delta_{s_{2}, s_{4}}, \mathcal{M}_{1}^{\prime}=\delta_{s_{1}, s_{4}} \delta_{s_{2}, s_{3}}, \mathcal{M}_{2}^{\prime}=\delta_{s_{1}, s_{2}} \delta_{s_{3}, s_{4}}
$$

Notice that the change of basis has only effected $\mathcal{M}_{2}$, leaving $\mathcal{M}_{0}$ and $\mathcal{M}_{1}$ unchanged. It is clear that all the arguments presented in the previous section are still applicable here. In fact, one can construct a cluster algorithm for the quantum XY model in this basis, which is the same as an algorithm for a quantum XZ model in the $\sigma^{3}$ basis. The cluster rules are given in figure 3 .

Also in the new basis the clusters are closed loops. However, now all the spins in a given cluster have the same orientation. Since now we are dealing with the XZ 

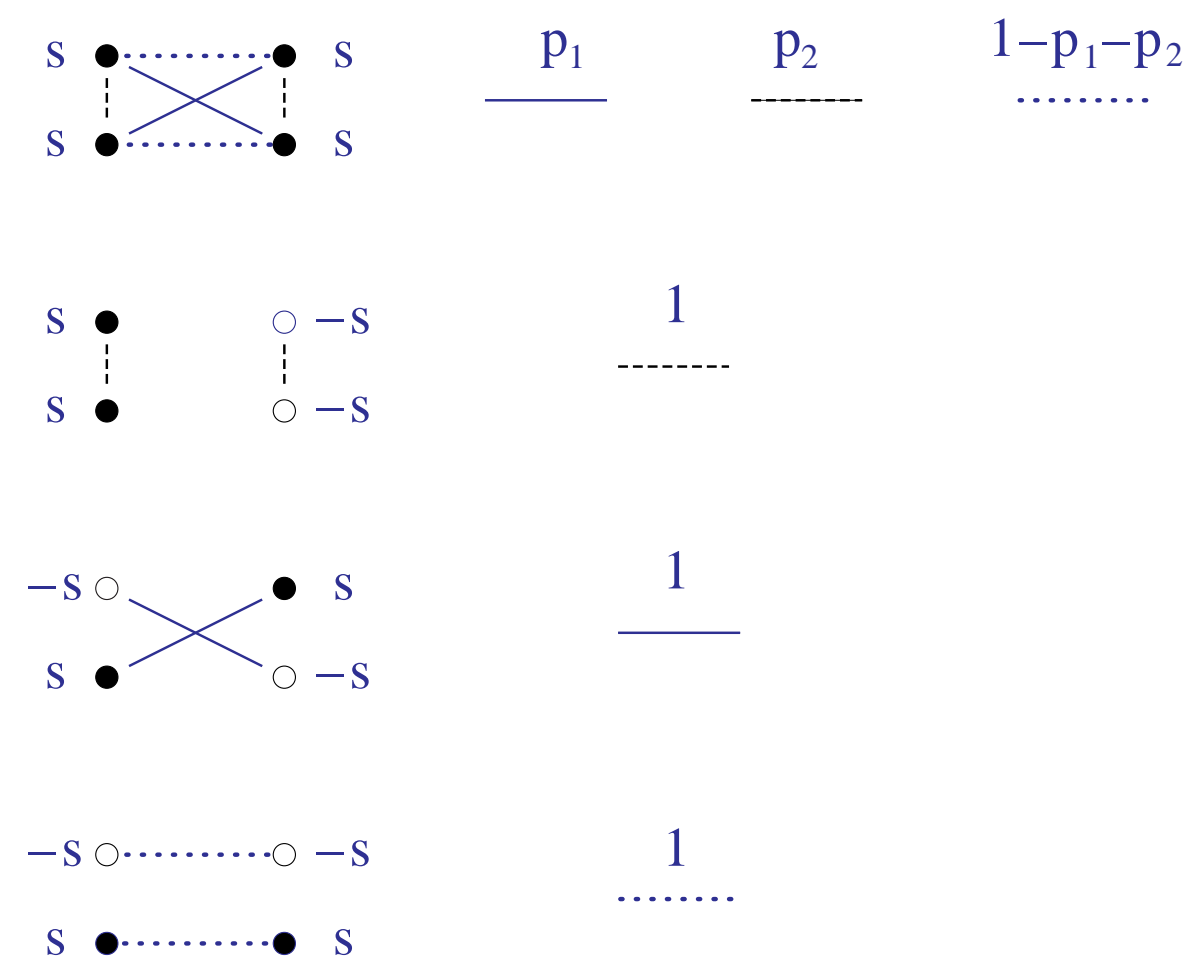

Figure 3: The rules for creating the bonds that define a graph $G$ in the $X Y$ model in the basis in which $\sigma^{2}$ is diagonal: The sites are described by filled circles and the time axis is vertical. The spin states in the $\sigma^{2}$ basis are denoted by $s$, which takes values \pm . The probabilities for choosing the bonds are given by $p_{1}=[1-\exp (-\epsilon J)] /[\exp (\epsilon J)+1]$ and $p_{2}=[1+\exp (-\epsilon J)] /[\exp (\epsilon J)+1]$.

model, the $\left\langle\sigma_{x}^{3} \sigma_{y}^{3}\right\rangle$ correlator must be the same as the $\left\langle\sigma_{x}^{2} \sigma_{y}^{2}\right\rangle$ correlator in the XY model. It is easy to see that, if $x$ and $y$ belong to different clusters, $\operatorname{Tr}\left[\sigma_{x}^{3} M_{\mathcal{C}_{i}}\right]$ is zero. However, when they belong to the same cluster, since all spins within a cluster have the same orientation, $\operatorname{Tr}\left[\sigma_{x}^{3} \sigma_{y}^{3} M_{\mathcal{C}_{i}}\right]=\operatorname{Tr}\left[M_{\mathcal{C}_{i}}\right]$. Thus, one gets a contribution 1 to $\left\langle\sigma_{x}^{3} \sigma_{y}^{3}\right\rangle$. This is exactly what we had in the previous basis for $\left\langle\sigma_{x}^{2} \sigma_{y}^{2}\right\rangle$. Note that in the previous basis one cannot show that $\left\langle\sigma_{x}^{3} \sigma_{y}^{3}\right\rangle$ gets a contribution 1 whenever $x$ and $y$ belong to the same cluster, since opposite spins can be present in the same cluster.

We have obtained numerical results that confirm the above conclusions. We have updated a $10 \times 10$ lattice with $N=100$ at $\beta J=1.0$ using both the $\mathrm{XY}$ and the $\mathrm{XZ}$ algorithm. The above arguments imply that both algorithms must yield identical cluster properties. The cluster size distribution has been measured with a single cluster algorithm. Figure 4 shows the histograms for the cluster size distributions obtained with the two algorithms. We see that they are identical within statistical errors. In table 1 we give the numerical values for the first few moments of the cluster size distributions of figure 4 . 


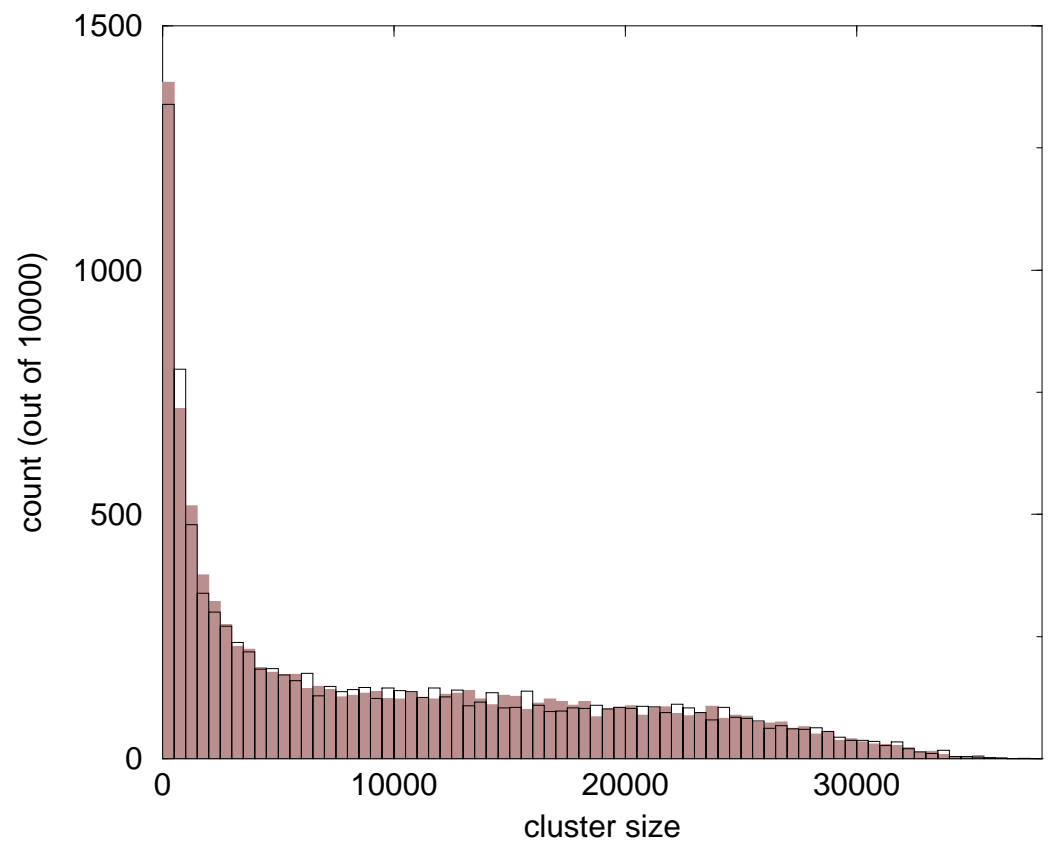

Figure 4: The histograms of cluster size distribution for the two algorithms discussed in the text: Both algorithms were run with single cluster updates. The data sets contain 10000 clusters each. The results of the XY algorithm are given by the shaded and that of the XZ algorithm are given by the unshaded histogram.

\section{Conclusions}

In this article we have developed a new method to evaluate any Green's function in quantum spin models using cluster algorithms. The method is based on the fact that clusters have a meaning independent of the basis. In other words, the construction of a cluster algorithm implies that one can rewrite the quantum spin model as a random cluster model in a basis-independent way. This is well-known in the case of the Potts model using the Fortuin-Kasteleyn mapping [9]. Here we have presented a simple argument showing that such a mapping also exists for quantum spin models. This mapping provides a new method to measure any operator once a cluster algorithm has been constructed. In this method a measurement of a certain operators reduces to the computation of traces in the Hilbert space for each individual cluster. In the quantum XY model, this turns out to be quite simple. The method applies to other models as well, and is useful for measuring quantities which could not be measured using standard methods.

The insight gained here extends easily to quantum link models, which were referred to in the introduction. Since these models are gauge invariant, the operators 


\begin{tabular}{|c|c|c|c|}
\hline Algorithm & $\langle\mathcal{C}\rangle$ & $\left\langle\mathcal{C}^{2}\right\rangle$ & $\left\langle\mathcal{C}^{3}\right\rangle$ \\
\hline $\mathrm{XY}$ & $9.750(120) \times 10^{3}$ & $1.790(33) \times 10^{8}$ & $3.941(96) \times 10^{12}$ \\
\hline $\mathrm{XZ}$ & $9.818(119) \times 10^{3}$ & $1.807(34) \times 10^{8}$ & $3.990(95) \times 10^{12}$ \\
\hline
\end{tabular}

Table 1: The first few moments of the cluster size for the two algorithms: The simulations were performed on a $10 \times 10$ lattice at $\beta J=1.0$ and $N=100$. This clearly demonstrates the basis-independence of the clusters.

of interest are closed Wilson loops. It seems difficult to construct cluster algorithms in the basis where the Wilson loops can be measured easily. For example, in the $U(1)$ quantum link model, a cluster algorithm has been constructed in an electric flux basis. The clusters resemble world-sheets of electric flux strings. When Gauss' law is imposed, the flux strings form closed loops and hence sweep out closed worldsheets. Although the Wilson loop operators are not diagonal in the electric flux basis, they can be computed using the method proposed in this article. The computation requires understanding the topology of the closed world-sheets generated by the Monte Carlo algorithm. For example, only if the cluster surface separates into two pieces when it is cut along the links belonging to the Wilson loop, there is a non-zero contribution to the operator. It is easy to show that Wilson loops get contributions from only one cluster at a time. At present, this is used to investigate the physics of the $U(1)$ quantum link model. The results of this study will be published elsewhere [8].

The connection with the random cluster model should be understood in more detail in the context of quantum cluster algorithms. One intriguing possibility is a solution of the negative sign problem, at least in a class of models. Perhaps the random cluster model suggests a natural basis to avoid negative signs. The new insight may also serve as a guide for constructing cluster algorithms for quantum link models with non-Abelian gauge groups.

\section{Acknowledgement}

One of the authors (S.C.) would like to thank the high energy theory group at the Fermi National Accelarator Laboratory, where part of this research was done, for its hospitality.

\section{References}

[1] F. Niedermayer, Cluster Algorithms, hep-lat/9704009

[2] H. G. Evertz, The Loop Algorithm, cond-mat/9707221. 
[3] R. Swendsen and J. -S. Wang, Phys. Rev. Lett. 58 (1987) 86.

[4] U. Wolff, Phys. Rev. Lett. 62 (1989) 361; Nucl. Phys. B 334 (1990) 581.

[5] H. G. Evertz, G. Lana and M. Marcu, Phys. Rev. Lett. 70 (1993) 875.

[6] U.-J. Wiese and H.-P. Ying, Z. Phys. B93 (1994) 147.

[7] S. Chandrasekharan and U.-J. Wiese, Nucl. Phys. B 492 (1997) 455;

R. Brower, S. Chandrasekharan and U.-J. Wiese, hep-th/9704106.

[8] B. B. Beard, R. Brower, S. Chandrasekharan, A. Tsapalis, U.-J. Wiese, work in progress.

[9] C. M. Fortuin and P. W. Kasteleyn, Physica 57 (1972) 536.

[10] N. V. Prekof'ev, B. V. Svistunov and I.S. Tupitsyn, cond-mat/9703200.

[11] B. B. Beard and U.-J. Wiese, Phys. Rev. Lett. 77 (1996) 5130.

[12] R. Brower and P. Tamayo, Phys. Rev. Lett. 62 (1989) 1087.

[13] N. Kawashima and J. E. Gubernatis, cond-mat/9502065. 